Itinéraires Itinéraires

Littérature, textes, cultures

\title{
Les femmes sadiennes avec Lacan
}

Juliette Feyel

\section{(2) OpenEdition}

\section{Journals}

Édition électronique

URL : https://journals.openedition.org/itineraires/683

DOI : 10.4000/itineraires.683

ISSN : 2427-920X

Éditeur

Pléiade

\section{Édition imprimée}

Date de publication : 1 mars 2014

Pagination : 73-84

ISBN : 978-2-343-02712-8

ISSN : 2100-1340

Référence électronique

Juliette Feyel, « Les femmes sadiennes avec Lacan », Itinéraires [En ligne], 2013-2 | 2014, mis en ligne le 01 novembre 2013, consulté le 21 septembre 2021. URL : http://journals.openedition.org/itineraires/ 683 ; DOI : https://doi.org/10.4000/itineraires.683

\section{$@ \oplus \Theta \Theta$}

Itinéraires est mis à disposition selon les termes de la licence Creative Commons Attribution - Pas d'Utilisation Commerciale - Pas de Modification 4.0 International. 


\section{Les femmes sadiennes avec Lacan}

\section{Résumé}

L'article utilise les concepts de la théorie lacanienne pour analyser la façon dont les personnages masculins et féminins fonctionnent les uns par rapport aux autres dans la fiction sadienne. Dans ses textes des années 1960, Lacan commente Sade en partant du principe que ses romans ne sont pas des utopies politiques. Il s'agirait plutôt d'une représentation dramatique des conflits animant la vie inconsciente. De plus, on propose de relire le diptyque de Justine et Juliette à la lumière de ce que Lacan expose sur la jouissance féminine dans son séminaire Encore. Les personnages d'hommes libertins seraient finalement pris dans un rapport asymétrique avec une instance féminine dédoublée.

Mots clés : Sade, Lacan, femmes, loi, jouissance

\section{Abstract}

The article proposes to use Lacanian notions to complicate our perception of men and women relationship in Sade's fiction. In his works of the early 1960s, Lacan comments on Sade, assuming that the novels are not to be taken as a political model but rather as a dramatic representation of the conflicts between the various agents of the unconscious. Moreover, applying the $20^{\text {th }}$ Seminar entitled Encore, On Feminine Sexuality to the two-part story of Justine and Juliette emphasizes the actual superiority of female characters over male libertines.

Keywords: Sade, Lacan, women, law, jouissance

L'œuvre de Sade scandalise à de multiples égards. Le cynisme avec lequel les libertins justifient les crimes les plus horrifiants n'en est qu'un parmi d'autres. Il est possible de trouver que Sade est subversif car il renverse toutes les conventions morales et libère la culture d'une tradition étouffante $^{1}$. Mais il est aussi possible de relever les signes d'une répartition traditionnelle des rapports de force et de faire de Sade le champion de

1. C'est ce sur quoi s'accordent Guillaume Apollinaire, André Breton, Pierre Klossowski et Georges Bataille. 
la tyrannie ${ }^{2}$. On a aussi souligné que, dans l'univers romanesque de Sade, c'était presque toujours la femme qui détenait le triste privilège de subir les assauts, les humiliations et les cruautés les plus extrêmes de libertins dont la virilité agressive et le désir de toute-puissance semblent ne pouvoir s'affirmer qu'aux dépens du sexe faible ${ }^{3}$. Cependant, à la tentation de répartir les deux sexes en deux classes étanches, celle des bourreaux et celle des victimes, certains personnages opposent leur irréductibilité. Comme le demande Annie Le Brun : " Pourquoi Juliette est-elle une femme ${ }^{4}$ ? » La psychanalyse pourrait-elle proposer une réponse à cette question en adoptant une autre méthode? Au lieu de faire une lecture politique des schémas actantiels sadiens, Lacan part de l'hypothèse que les personnages incarnent les différentes instances de l'inconscient. À partir d'un tel postulat, la fiction sadienne nous apparaît moins comme le microcosme d'une société utopique que comme le théâtre des conflits animant la vie psychique.

Lacan commente l'œuvre de Sade lorsqu'il écrit L'Éthique de la

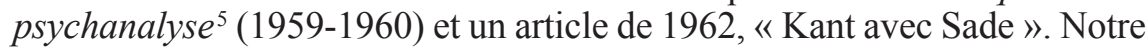
but ne sera pas d'étudier les modalités de la réception de Sade par Lacan, un travail qui a déjà été minutieusement développé par Éric Marty ${ }^{6}$. Nous proposons de parcourir le même chemin mais dans le sens du retour, en faisant l'expérience de lire Sade « avec » Lacan.

Dans ses écrits sur Sade, Lacan ne s'exprime quasiment pas sur la question des personnages féminins. Il raffine cependant considérablement sa compréhension de la fonction féminine dans un séminaire de 1972-1973, Encore 7 , mais cette fois, il ne revient pas au corpus romanesque du divin marquis. Pour voir comment la théorie lacanienne peut éclairer le fonctionnement des rapports entre les sexes chez Sade, il faudra donc essayer de saisir au vol les allusions ébauchées dans les années 1960, prendre en compte la façon dont la notion de «jouissance » a évolué jusque dans les années 1970 et tirer les fils de textes disparates afin de construire par extrapolation un texte que Lacan n'a pas écrit. Ce que ce texte aurait pu être, nous allons tenter ici de l'imaginer.

\section{Le Nom du Père et le corps interdit de la Mère}

L'Éthique de la psychanalyse se présente comme une longue comparaison ayant pour but de mettre en valeur les points communs de l'œuvre la plus immorale avec celle qui fonde la morale :

2. C'est la position défendue par Albert Camus, Theodor Adorno et Max Horkheimer, par exemple.

3. Angela Carter, The Sadeian Woman, Londres, Virago, 1979.

4. Annie Le Brun, On n'enchaîne pas les volcans, Paris, Gallimard, 2006, p. 127.

5. Jacques Lacan, L'Éthique de la psychanalyse, Séminaire VII, Paris, Seuil, 1986 et Écrits, Paris, Seuil, 1966, p. 765-790.

6. Éric Marty, Pourquoi le XXe siècle a-t-il pris Sade au sérieux?, Paris, Seuil, 2011, p. 171-267.

7. Jacques Lacan, Encore, Séminaire XX, Paris, Seuil, 1975. 
Si la Critique de la raison pratique est parue en 1788, sept ans après la première édition de la Critique de la raison pure, il est un autre ouvrage qui, lui, est paru six ans après la Critique de la raison pratique, un peu au lendemain de Thermidor, en 1795, et qui s'appelle La Philosophie dans le boudoir ${ }^{8}$.

Tout comme la morale pure kantienne, l'antimorale sadienne est un formalisme; elle s'appuie sur un impératif catégorique dont le caractère obligeant dépend de son universalité. Kant affirme que l'un des critères de la moralité consiste en son détachement de toute "pathologie», c'est-à-dire de tout affect qui pourrait empêcher la raison pratique d'appliquer la loi avec rigueur. Sade montre les libertins obéissant à une discipline ascétique destinée à leur faire atteindre un état d'《 apathie » nécessaire à la jouissance. Qu'ajoute finalement Sade au kantisme? Il lui confère un contenu phénoménal. Il a su prolonger la logique kantienne en donnant figure à l'objet de la loi morale. Le libertin qui torture ses victimes sans pitié illustre l'application fidèle de la Loi du désir. Ce déplacement de la théorie vers la scène du fantasme - de phantasma, « image »-, autrement dit cette fictionnalisation, révèle dans toute sa clarté ce que le discours théorique avait masqué, à savoir l'insoutenable cruauté qui découle de l'application rigoureuse des lois.

Poursuivant sa comparaison, Lacan établit des parallèles entre plusieurs couples conceptuels. Là où Kant distingue le Bien - das Gute - en tant que valeur, du bien(-être) - wohl - et souligne que le respect du Gute doit être " apathétique », Sade distingue deux morales, celle du libertinage apathique et celle de la société. Lacan amène ces couples à recouper celui du désir de jouissance opposé au principe de plaisir. Le principe de plaisir est ce que le sujet recherche pour autant qu'il supprime une tension désagréable et permet au moi de maintenir son intégrité. Ce principe de plaisir seconde la castration à laquelle le sujet a été soumis lors du complexe d'Edipe, lorsque le désir d'inceste a été interdit et que le moi s'est psychiquement détaché du corps de la Mère. Lacan joue sur l'homophonie Non/Nom du Père pour indiquer que la construction du sujet comme être séparé s'opère par la médiation du symbolique, c'est-à-dire du langage.

L'Éthique de la psychanalyse montre que la civilisation repose sur des interdits civilisateurs dont le Deutéronome fournit un illustre exemple. Lacan désigne la loi sociale par le Nom du Père car, dans un geste inaugural, le Père mythique fondateur interdit, tranche, sépare. Sade a la même intuition lorsque, dans «Français, encore un effort si vous voulez être républicains ", il isole quatre crimes cardinaux violant tout ce qui fonde les règles sociales : la calomnie, le vol, l'impureté et le meurtre 9 . Au fond, la Loi paternelle garantit l'ordre, le propre, la différenciation; il hisse des

8. Jacques Lacan, L'Éthique, op. cit., p. 95.

9. Sade, La Philosophie dans le boudoir [1795], dans Euvres, éd. Michel Delon, Paris, Gallimard, coll. « Bibliothèque de la Pléiade », t. III, 1998, p. 125. 
remparts contre le chaos, le mélange, la confusion. Sade évoque la mise en commun des femmes car, bien avant Lévi-Strauss ${ }^{10}$, il a senti que la prohibition de l'inceste et le mariage canalisaient le désir et participaient de ce qui rendait la vie sociale possible. À l'échelle de l'individu, le Nom du Père est perçu comme un surmoi inhibiteur, culpabilisant, qui joue sur le principe de plaisir pour réguler les pulsions et modérer le désir ${ }^{11}$. Il force le moi à respecter des limites car elles le protègent et le soutiennent.

Que se passera-t-il si on « ouvre toutes grandes les vannes [que Sade] propose imaginairement à 1 'horizon du désir ${ }^{12}$ »? Le principe de plaisir régule la vie sociale tandis que le désir renverse les digues érigées par le Nom du Père. Un affranchissement intégral conduirait à une crue, à un état d'indistinction insoutenable dans lequel le moi, s'il venait à en jouir, serait anéanti. Ce territoire au-delà de la Loi paternelle, Lacan le nomme l'Autre de la Loi. Fantasmatiquement, il est associé au corps interdit de la Mère, à cette continuité originelle dont le moi a été séparé.

Dans «Français, encore un effort », l'Autre archaïque maternel ordonne au libertin de transgresser toutes les lois, fût-ce aux dépens d'autrui. Notons bien que cette injonction prend la forme universelle d'une loi. Aussi s'impose-t-elle autant que la Loi paternelle. L'Autre agit comme un deuxième surmoi qui persécute le moi : «Le surmoi, c'est l'impératif de la jouissance - Jouis ${ }^{13}$ ! » Le moi est donc tiraillé entre deux commandements antithétiques qui s'excluent : d'un côté le Nom du Père qui maintient la castration et soutient l'instinct de conservation du moi; de l'autre, l'impératif de jouir émanant de l'Autre. Sade dénonce la pusillanimité de la Loi du Père, d'une morale raisonnable, soumise au pathologique. « Français, encore un effort » révèle la face cachée de l'injonction à être libre, le caractère insoutenable d'un désir parfaitement affranchi. Au-delà du principe de plaisir se profile la cruauté de la jouissance.

\section{La triangulation du rapport sexuel}

En ce qui concerne le rapport sexuel, Lacan souligne dans ses textes des années 1970 la complexité de l'instance féminine dans l'inconscient. Rejetant le rêve d'une complémentarité entre homme et femme que le mythe de l'androgyne met en scène, Lacan affirme que dans un premier temps, « la femme n'entre en fonction dans le rapport sexuel qu'en tant que la mère ${ }^{14} \gg$. Le rapport du moi masculin au féminin est triangulaire car le féminin occupe deux pôles à la fois. Il est à la source du désir, en tant

10. Claude Lévi-Strauss, Les Structures élémentaires de la parenté, Paris, PUF, 1949.

11. Sur ce point, Lacan ne dévie pas de ce que dit Freud de la fonction sociale du surmoi. Il modère les pulsions libidinales des individus dans Malaise dans la civilisation (1929).

12. Jacques Lacan, L'Éthique, op. cit., p. 96.

13. Jacques Lacan, Encore, op. cit., p. 10.

14. Ibid., p. 36. 
qu'il renvoie fantasmatiquement à l'Autre maternel archaïque ; il est également l'objet qui suscite le désir, horizon auquel le sujet aspire et que Lacan appelle la Chose. La femme est à la fois origine et finalité, cause et objet du désir. Cette relation triangulaire apparaît dans Les Cent Vingt Journées de Sodome. Deux types de femmes entourent les libertins de sexe masculin : d'un côté les victimes qui subissent leurs assauts, de l'autre les historiennes qui prescrivent aux libertins de jouir.

À l'un des deux pôles, la Chose est issue d'une ruse de l'Autre pour séduire le libertin. L'Autre, cause réelle du désir, est indistinction. Il ne peut donc rien soutenir et ne susciterait que l'horreur chez le moi s'il n'était masqué. Il faut donc qu'il propose un objet pour soutenir le désir. Michel Delon ${ }^{15}$ note que la beauté de la victime est toujours marmoréenne, délicatement ciselée comme la statuaire antique, blanche, nettement séparée. C'est que l'être délimité par le Nom du Père représente une provocation pour le moi castré; Loi et désir de transgression s'impliquent mutuellement. Comme le dit Lacan : « Je n'ai eu connaissance de la Chose que par la Loi. En effet, je n'aurais pas eu l'idée de la convoiter si la Loi n'avait dit - Tu ne la convoiteras pas ${ }^{16}$ ». Justine n'occupe la place de l'objet du désir, n'est « intéressante » que pour autant qu'elle semble inviolée et inviolable. À sa beauté s'ajoutent sa pudeur et son innocence, qui la rendent encore plus désirable. La virginité de Justine perdure au-delà de la vraisemblance empirique car c'est de son héroïne et de la convoitise qu'elle inspire que se soutient le fantasme mis en scène par le roman : "Le point d'aphanasis, supposé en [symbole du sujet castré], doit être dans l'imagination indéfiniment reculé ${ }^{17} \gg$.

À l'autre pôle, la nature occupe dans l'ensemble des romans le même emploi que les historiennes des Cent Vingt Journées. Le fait que Sade remplace l'idée de Dieu par la nature a deux conséquences. D'abord, la nature est une instance féminine. Ensuite, c'est une entité impersonnelle. La nature en tant que continuité indistincte, dynamique inlassable de décomposition et de recomposition des individus, possède les qualités de l'Autre. Lacan l'évoque à travers des métaphores liquides : « Sa nature de cascade, ces buffets d'eau du désir édifiés pour qu'elle irise [...] volupté baroque $[\ldots]$ ce qui est là ruisselant ${ }^{18} \gg$. Cette puissance aveugle qui fait horreur, à la fois pourvoyeuse de vie et donneuse de mort, Sade lui donne les traits de la lave volcanique. Au bout de son initiation au libertinage, Olympe Borghèse est jetée dans la fournaise du Vésuve, anéantie dans un flamboiement terrible. Formidable marâtre que cette nature sadienne puisqu'elle met au monde des enfants pour les harceler de maux et leur ôter finalement la vie, comme en témoigne également le sort de Justine.

15. Michel Delon, «L'obsession anale de Sade», Annales historiques de la Révolution française, $\mathrm{n}^{\circ}$ 361, 2010, p. 131-144.

16. Jacques Lacan, L'Éthique, op. cit., p. 101.

17. Jacques Lacan, « Kant avec Sade » [1962], dans Écrits, op. cit., p. 775.

18. Ibid., p. 786. 
Les deux versions de «Kant avec Sade » témoignent d'une précision apportée entre 1960 et 1962 . L'impératif sadien de la jouissance, le « droit à jouir d'autrui quel qu'il soit ${ }^{19}$ », est reformulé ainsi : «J'ai le droit de jouir de ton corps, peut me dire quiconque, et ce droit, je l'exercerai, sans qu'aucune limite m'arrête dans le caprice des exactions que j'aie le goût d'y $\operatorname{assouvir}^{20} \gg$. De même que Kant a distingué l'auteur et l'exécutant de la Loi, Lacan formule l'impératif à travers une double énonciation qui montre que le libertin obéit à une Loi dont l'auteur réel est une voix anonyme. Il ne fait pour Lacan pas de doute que cette voix est celle de l'Autre. Le libertinage serait commandé par le désir de jouir de l'Autre. Effectivement, le libertin ne cesse de justifier ses actes en se référant à la nature. Irresponsable, il est agi par cette entité qui le dépasse, dont il ne peut percer les desseins et qui se sert de lui pour exercer sa puissance meurtrière :

Tourmentez donc anéantissez, détruisez, massacrez, brûlez, pulvérisez, fondez, variez enfin sous cent mille formes toutes les productions des trois règnes, vous n'aurez jamais fait que les servir, vous n'aurez fait que leur être utile. Vous aurez rempli leurs lois, vous aurez accompli celles de la nature $[\ldots]^{21}$.

C'est donc moins le libertin que l'Autre qui veut jouir et qui persécute les victimes : «C'est ce qu'il advient de l'exécuteur dans l'expérience sadique, quand sa présence à la limite se résume à n'en être plus que l'instrument ${ }^{22} \gg$.

Il n'est toutefois pas dit que le libertin accepte son sort. Dans une longue protestation contre la nature, Almani formule le vœu de contrer cette maîtresse en retournant sa puissance destructrice contre elle :

Je l'imiterai mais en la détestant; je la copierai; elle le veut, mais ce ne sera qu'en la maudissant [...] furieux de voir que mes passions la servent [...] barrons-la dans ses œuvres pour l'insulter plus vivement; et troublonsla dans ses œuvres pour l'outrager plus sûrement ${ }^{23}$.

Nombreux sont les épisodes où la cruauté des libertins se déchaîne contre les mères. Dans l'histoire de Brisa-Testa, le père cherche à exercer un contrôle total sur ses enfants. Il les force à se faire les instruments apathiques de sa volonté en les amenant à déshonorer puis à détruire leur propre mère. Cette sommation et son obsession pour l'inceste illustrent une tentative d'usurpation de l'autorité maternelle par le père. Ce dernier cherche à se substituer à l'auteur de la loi commandant de jouir.

19. Jacques Lacan, L'Éthique, op. cit., p. 96.

20. Jacques Lacan, « Kant avec Sade », op. cit., p. 768-769 (nous soulignons).

21. Sade, Histoire de Juliette ou les Prospérités du vice, dans CEuvres, op. cit., t. III, p. 878.

22. Jacques Lacan, « Kant avec Sade », op. cit., p. 773.

23. Sade, La Philosophie dans le boudoir, op. cit., p. 779. 
Le libertin rêve de doubler la nature par sa puissance, de surpasser son flot continu, créateur et destructeur. Il exhibe les dimensions prodigieuses de son phallus et accomplit des prouesses physiques extravagantes. Il prétend, comme Almani, éteindre avec son sperme la lave des volcans ${ }^{24}$. Le phallus étant aussi défini par Lacan comme signifiant - substitut - de la jouissance, il serait possible d'interpréter l'inflation verbale et les interminables dissertations des libertins comme une façon pour le sujet masculin de combler la béance de l'Autre par le phallus du Père.

Mais cette aspiration est vaine, le libertin reste impuissant face au désir sans fond de l'Autre qui répète inlassablement Encore. Almani doit reconnaître : « La putain s'est moquée de moi, ses ressources l'emportaient sur les miennes : nous luttions trop inégalement ${ }^{25} \gg$. Là où règne l'Autre, ne règne que le silence; la virtuosité verbale du libertin n'a d'égale que la vanité de sa parole puisqu'elle doit fatalement s'interrompre quand le livre s'achève. L'aphanasis succède toujours à la jouissance phallique. D'après Lacan, « la jouissance phallique est l'obstacle par quoi l'homme n'arrive pas, dirai-je, à jouir du corps de la femme, précisément parce que ce dont il jouit, c'est de la jouissance de l'organe ${ }^{26}$ ».

Impuissant, le libertin peut choisir de nier le désir de l'Autre. Par le pouvoir de la parole, Dolmancé s'impose en seul maître d'Eugénie et cherche, à travers elle, à arrêter le flot destructeur de la nature en ordonnant que soit cousue la voie par laquelle la Mère archaïque donne naissance à ses martyrs : «Écartez vos cuisses, maman, que je vous couse, afin que vous ne me donniez plus ni frères ni sœurs ${ }^{27} \gg$. Pour Lacan, qui évoque ce passage, le libertin est un pervers car il refuse de reconnaître la béance au cœur de l'Autre désirant. Ce faisant, il reste du côté du Nom du Père, il est « père-vers ». La suture de la béance de l'Autre conduit Lacan à trouver Sade bien pusillanime. Il n'a pas su être à la hauteur de l'injonction de « Français, encore un effort ». Il a reculé face au désir sans fond de l'Autre et s'est réfugié sous la protection du principe de plaisir : « Notre verdict est confirmé sur la soumission de Sade à la $\operatorname{Loi}^{28}$ »).

Au regard de ses autres textes, le verdict de 1962 semble un peu trop sévère. Il se montrait beaucoup plus admiratif dans L'Éthique de la psychanalyse. Que s'est-il passé? Cela ne masquerait-il pas une autre critique adressée, celle-ci, à ses contemporains? Car Lacan ne cache pas son agacement vis-à-vis de ceux qui, comme Klossowski, érigent le divin marquis en héros révolutionnaire. En accusant injustement Sade de manquer d'humour,

24. Sade, Histoire de Juliette, op. cit., p. 780.

25. Ibid., p. 779.

26. Jacques Lacan, Encore, op. cit., p. 13.

27. Sade, La philosophie dans le boudoir, op. cit., p. 175.

28. Jacques Lacan, « Kant avec Sade », op. cit., p. 790. 
ne s'en prend-il pas plutôt au sérieux de ses contemporains, à « l'invasion pédantesque qui pèse sur les lettres françaises depuis la W.W.II. ${ }^{29}$ »?

De plus, ce verdict confond le libertin avec Sade, le pervers de la fiction avec le romancier, comme si Lacan avait changé de méthode herméneutique. Si l'on part du principe que le roman déroule le fantasme inconscient du désir, l'homme libertin exécute ce que l'Autre lui impose et ne peut donc, pas plus que les autres personnages, revendiquer le statut de 1'《 auteur».

En outre, Lacan oublie que la fiction se soutient d'un objet substitutif. En prétendant couvrir le silence, elle nie le désir de l'Autre. Le texte littéraire est suture, pervers a priori. Sade pusillanime, Sade soumis à la Loi ? Ne serait-ce pas plutôt le roman, tissu de signifiants, qui demeurerait soumis au Nom du Père? Comment alors atteindre, par le recours au signifiant, l'Autre des signifiants?

\section{Justine et Juliette ou l'évolution du diptyque sororal}

Après 1962, Lacan n'écrit plus sur Sade et s'intéresse de plus en plus aux extases des mystiques, à Sainte Thérèse, à Angèle de Foligno. Dans Encore, il est amené à distinguer deux jouissances. Tandis que l'homme reste du côté de la jouissance phallique, c'est-à-dire qu'il demeure enfermé du côté du Nom du Père et ne peut jouir que dans l'ordre des signifiants - du phallus -, la femme n'est « pas toute ${ }^{30} »$. Entendons : elle n'est pas castrée, séparée de la Mère de la même manière que le sujet masculin, une part d'elle reste irréductible à l'emprise du Nom du Père. De cette part qui jouit, elle ne peut rien dire car elle échappe au symbolique, c'est ce qu'il désigne du nom d'autre jouissance - jouissance de l'Autre. Si Lacan, peut-être pour les raisons que nous avons évoquées, a critiqué durement La Philosophie dans le boudoir, roman du Père précepteur et prescripteur, un réexamen de l'histoire des deux sœurs à la lumière des découvertes sur les mystiques aurait peut-être pu l'amener à réajuster son jugement sur Sade. Ce réexamen, essayons de l'imaginer.

$\mathrm{Au}$ gré des remaniements du récit des aventures des deux sœurs, le personnage de Juliette prend de plus en plus d'ampleur. Du statut de faire-valoir, son histoire devient progressivement plus volumineuse, au point de devenir autonome et d'égaler les dimensions de l'histoire de Justine. Seule la dernière réécriture fait apparaître un vrai diptyque. Ce processus de méiose textuelle permet d'assister à un raffinement des fonctions de chaque personnage. La fonction de narratrice dévolue à Justine dans les deux premières versions est confiée à Juliette. Alors que sujet et objet de la narration étaient fondus en un seul personnage, l'importance croissante de Juliette coïncide avec le choix d'une narration à la troisième personne pour

29. Ibid., p. 788.

30. Jacques Lacan, Encore, op. cit., p. 13. 
La Nouvelle Justine. Le résultat est une distinction plus nette entre auteur et patient du récit, entre le bourreau et la victime, entre l'Autre qui désire jouir et la Chose dont on jouit.

En étant une femme, Juliette se voit dévolu le rôle de l'Autre. C'est par sa voix que sont décrites les débauches et elle ne tarde pas à devenir maître dans l'art de les prescrire. Ses prospérités découlent de son immoralisme mais surtout de sa créativité, ce dont les libertins ne cessent de la louer : «Oh! sacredieu, Juliette, quelle imagination bizarre t’a donnée la nature $^{31}$ ! » Douée d'une affinité particulière avec la nature, précisément parce qu'elle n'est « pas toute », Juliette inspire aux libertins les crimes dont ils se font les instruments. Ajoutons que, non contente d'inspirer les crimes, elle continue à encourager les criminels durant leurs débauches, comme s'il fallait veiller à ce que leur apathie ne faiblisse pas : « Je l'excite par des $\operatorname{propos}^{32} »$. Au fur et à mesure de sa formation, Juliette parvient à s'identifier à la nature dans un moment de fureur érotique où, atteignant l'apathie, elle éteint définitivement les sentiments qui l'attachent à sa fille et la livre non seulement à Noirceuil mais surtout au feu. Elle incarne désormais pour de bon le rôle de la Mère archaïque destructrice.

Mais pour parachever cette identification avec l'Autre, il faudrait cependant que Juliette se sacrifie elle-même et se fonde à son tour dans le feu liquide de la jouissance. Cela démentirait Lacan qui soulignait un manque chez Sade :

L'œuvre jamais ne nous présente le succès d'une séduction où pourtant se couronnerait le fantasme : celle par quoi la victime, fût-ce en son dernier spasme, viendrait à consentir à l'intention de son tourmenteur, voire s'enrôlerait de son côté par l'élan de ce consentement ${ }^{33}$.

Juliette s'enrôle, mais elle ne va pas jusqu'à s'offrir en victime consentante, elle ne va pas jusqu'au bout du désir de l'Autre.

C'est à Justine qu'est confiée cette mission. Dans Les Infortunes de la $v e r t u$, sa candeur peut la rendre parfois ridicule, comme c'est le cas dans la forêt où Bressac la fait attacher à un arbre et où elle ne comprend pas ce qui se passe derrière elle. En revanche, Les Malheurs de la vertu présente une Justine trop avide de se justifier, trop empêtrée dans une rhétorique de la mauvaise foi pour ne pas être suspecte. Cherchant les moyens d'éviter la mort à Mme de Bressac, elle écarte la solution qui semblerait la plus efficace : « Il me restait la voie de la justice, mais rien au monde n'aurait pu me résoudre à la prendre ${ }^{34} »$. Elle n'est pas dépourvue d'esprit, malgré ce que prétend le narrateur, en témoignent l'éloquence de ses discours édifiants

31. Sade, Histoire de Juliette, op. cit., p. 1257.

32. Ibid., p. 1248.

33. Jacques Lacan, « Kant avec Sade », op. cit., p. 787.

34. Sade, Justine ou les Malheurs de la vertu, dans Euvres, éd. Michel Delon, Paris, Gallimard, coll. « Bibliothèque de la Pléiade », t. II, 1995, p. 193. 
et les calculs auxquels elle se livre très rapidement afin de trouver le plus sûr moyen d'échouer à sauver sa maîtresse : " Cette réflexion, qui fut en elle l'ouvrage d'un instant ${ }^{35} \ldots$.. ». Elle se montre capable de duplicité : « Comme un retour si prompt l'eût infailliblement fait soupçonner de fraude, elle ménagea quelque temps sa défaite, et mit Bressac dans le cas de lui répéter souvent ses maximes ${ }^{36} \gg$. Le changement d'énonciation permet au narrateur hétérodiégétique de se livrer à sa verve ironique. Confrontée aux caprices de Bressac, Justine n' exprime que de faibles protestations et se contente de pleurer. Le narrateur insiste trop lourdement sur sa naïveté pour qu'on ne doute pas de la sincérité de ces larmes :

Elle ne connaissait pas le monstre auquel elle avait affaire; elle ne savait pas, l'innocente créature, à quel point les passions étayent et fortifient le crime dans une âme telle que celle de Bressac; elle ignorait que tout ce que la vertu, la sensibilité peuvent inspirer dans pareille circonstance, devient, dans le cœur du scélérat, comme autant d'aiguillons ${ }^{37}$.

Justine semble en fait très bien connaître la dialectique de la Loi et du désir; Madame de Bressac sait discerner en elle « cet âge naïf où la vertu séduit et trompe à la fois les hommes ${ }^{38} »$. De nombreux indices forcent à reconnaître la secrète complicité de cette victime avec son bourreau réel, l'Autre.

Justine obéit mieux que tous les autres personnages à l'impératif de la jouissance. Reprenons la formulation de 1962. L'exécutant de la Loi de l'Autre pourrait être le libertin mais pourrait aussi bien être Justine : « J'ai le droit de jouir de ton corps, peut me dire quiconque... » Elle s'expose à tout, s'offre à tout, quiconque peut user d'elle et c'est bien ce que Bressac lui envie lorsqu'il lui décrit son désir le plus cher : «Qu'il est délicieux d'être la catin de tous ceux qui veulent de vous ${ }^{39} \gg$. Il est d'ailleurs intéressant de rapprocher cette phrase de ce que Mme Delbène enseigne à Juliette :

Tu verras la mutine, ravie d'avoir été violée, s'assouplissant sous tes désirs nerveux, venir d'elle-même s'offrir à tes fers... te présenter les mains pour que tu la captives; devenue ton esclave au lieu d'être ta souveraine, elle enseignera finement à ton cœur la façon de l'outrager encore mieux, comme si elle se plaisait dans l'avilissement, et comme si ce n'était réellement qu'en t'indiquant de l'insulter à l'excès qu'elle eût l'art de te mieux réduire à ses lois ${ }^{40}$.

Juliette fait son apprentissage de libertine en se préparant à servir celle dont elle devra se montrer digne et Justine est finalement la seule à se montrer à la hauteur de la morale apathique du désir, à avoir compris comment accéder le plus sûrement à la jouissance.

35. Ibid., p. 509.

36. Ibid.

37. Ibid., p. 508.

38. Ibid., p. 494.

39. Ibid., p. 181.

40. Sade, Histoire de Juliette, op. cit., p. 194. 
Le passage où Justine se livre à la volupté du moine débauché comporte une forte dose d'ambiguité; elle demeure « immobile, fermement persuadée que tout ce qu'on lui fait n'a d'autre but que de la conduire pas à pas vers la perfection céleste ${ }^{41} \ldots »$. Pris au sens littéral, cela signifierait que le libertin, en la trompant, en la violant, se ferait l'auxiliaire de l'accès de Justine à un au-delà du plaisir. Lacan relie la jouissance de l'Autre avec l'expérience des mystiques et se réfère à la célèbre statue du Bernin représentant Sainte Thérèse - Thérèse, l'un des noms de Justine. La perfection céleste, Justine finit par l'atteindre quand Sade la fait périr dans une véritable transverbération : « La foudre, entrée par la bouche, était sortie par le vagin : d'affreuses plaisanteries sont faites sur les deux routes parcourues par le feu du $\operatorname{ciel}^{42} »$. Les libertins, pervers, croient y voir la défaite de la vertu et la confirmation de leur triomphe. Le « feu du ciel » masculin aurait apposé la marque du phallus sur le corps féminin, sur la Chose. Ils oublient qu'en réalité c'est la nature qui jouit dans l'anéantissement de Justine. La foudre a ouvert son corps de part en part, en a détruit les limites. Consumée par le feu, l'héroïne s'abîme dans la continuité volcanique de la jouissance de l'Autre.

Dans Les Infortunes et Les Malheurs, Juliette prend le voile après avoir assisté à la mort de sa sœur. Le narrateur laisse perfidement croire qu'il s'agit d'un renoncement au libertinage et à la jouissance, c'est-à-dire d'une conversion à la loi du Père. Mais à quelle règle Juliette se convertitelle vraiment après avoir comparé son destin à celui de sa sœur? Il se trouve que l'ordre qu'elle choisit, celui des Carmélites, est celui de Sainte Thérèse.

Dans la troisième version, Juliette n'a pas besoin de se convertir pour connaître les mêmes félicités que Justine. Une structure est mise en place dès le début pour suggérer leur secrète identité. L'orgasme de Juliette se superpose avec les pleurs de Justine. Excrétions du haut et excrétions du bas proviennent de la même « source » puisque la jouissance de Juliette « tarit la source des larmes ${ }^{43}$ ». L'équivalence semble parfaite dans cette équivoque expression : «[...] son jeune foutre, éjaculé sous les yeux baissés de la vertu ${ }^{44} \ldots$.. Les larmes seraient issues d'un déplacement, comme dans le travail du rêve, des flux de la jouissance vers une autre partie du corps. L'indice nous est donné dès le début de l'histoire et les parcours diamétralement opposés des deux jeunes filles renvoient l'un à l'autre comme les lignes de fuite d'une parfaite symétrie. Comme dans un miroir, chacune peut mirer l'autre partie d'elle-même dans les aventures de sa sœur. Et si Juliette occupe la place de l'Autre, si Justine occupe la place de la Chose, rappelons que Lacan tend parfois à les confondre : «En réalité il doit être posé comme extérieur, ce das Ding, cet Autre préhistorique impossible à oublier ${ }^{45} »$.

41. Sade, Justine ou les Malheurs de la vertu, op. cit., p. 596.

42. Sade, Histoire de Juliette, op. cit., p. 1259.

43. Sade, La Nouvelle Justine, dans CEuvres, op. cit., t. II, p. 398.

44. Ibid.

45. Jacques Lacan, L'Éthique, op. cit., p. 87. 
Les deux facettes de la même médaille se rejoignent et une fois que Justine est foudroyée, Juliette ne tarde pas à disparaître tout bonnement du texte.

Les dernières lignes du diptyque surprennent car, in extremis, le narrateur réduit à néant la somme des efforts mis en œuvre pour raconter l'histoire des deux sœurs.

[Juliette] enlève absolument à tout écrivain la possibilité de la montrer au public. Ceux qui voudraient l'entreprendre ne le feraient qu'en nous offrant leurs rêveries pour des réalités, ce qui serait d'une étonnante différence aux yeux des gens de goût, et particulièrement de ceux qui ont pris quelque intérêt à la lecture de cet ouvrage ${ }^{46}$.

Le texte dénonce son impuissance et suggère un ailleurs qu'il ne pourrait évoquer que par le détour de piètres illusions, de rêveries, de fantasmes. L'essentiel de la jouissance reste finalement au-delà des mots, de même que le sujet soumis au Nom du Père ne goûte jamais à la jouissance de 1'Autre. Le roman serait alors la Chose qui masque la béance de l'Autre. Le lecteur qui ne verrait pas se profiler l'Autre au-delà du bord du texte commettrait la même erreur que les libertins. Se livrant à de derniers outrages sur la partie du corps de Justine qui réveillait toutes les convoitises, croyant posséder absolument la Chose, les libertins fétichisent la Chose et ignorent que l'objet réel du désir est la jouissance.

En proposant de considérer les romans sadiens comme des projections sur la scène de l'imagination des conflits internes de la psyché, Lacan propose un éclairage original sur le fonctionnement des personnages féminins et masculins. Relire la fiction sadienne à la lumière de la théorie développée par Lacan permet de remettre en question une organisation duelle du schéma actantiel. Le sujet masculin se voit pris dans un rapport sexuel triangulaire, face à deux instances féminines : d'un côté l'Autre qui ordonne de jouir, de l'autre la Chose qui suscite le désir et s'expose au pouvoir destructeur de la jouissance. Exécuteur d'une loi qui le dépasse, l'homme libertin en serait réduit à manquer (à) l'Autre. En le niant, il resterait soumis au phallus et au règne des signifiants, il resterait attaché au Nom du Père, prisonnier de la dialectique de la Loi et de la transgression. De l'autre côté des limites du plaisir, les parcours de Justine et de Juliette représenteraient la complicité de la victime avec son bourreau dans le flamboiement d'un désir de jouissance poursuivi jusqu'au bout.

Juliette Feyel

Fitzwilliam College, Université de Cambridge

46. Sade, Histoire de Juliette, op. cit., p. 1262. 